\title{
A Bounce-Averaged Kinetic Model of the Ring Current Ion Population
}

\author{
V. K. Jordanova,* J. U. Kozyra,* G. V. Khazanov,* A. F. Nagy,* \\ C. E. Rasmussen,* and M.-C. Fok**
}

Abstract. A bounce-averaged ring current kinetic model for arbitrary pitch angle, including losses due to charge exchange and Coulomb collisions along ion drift paths, is developed and solved numerically. Results from simplified model runs, intended to illustrate the effects of adiabatic drifts and collisional losses on the proton population, are presented. The processes of i) particle acceleration under the conditions of time-independent magnetospheric electric fields; ii) a predominant loss of particles with small pitch angles due to charge exchange; and iii) a buildup of a low-energy population caused by the Coulomb drag energy degradation, are discussed.

\section{Introduction}

The terrestrial ring current is associated with the motion of energetic charged particles, governed not only by the magnetospheric convection and corotation electric fields, but also by the gradient and curvature of the geomagnetic field. The trajectories of these particles have been studied during different magnetospheric conditions using adiabatic drift theory [e.g. Ejiri, 1978; Takahashi and Iyemori, 1989]. Such investigations are very useful in order to explain not only the process of formation of the storm-time ring current, but also some features in the measured energy spectra of the ring current ion species like dips in different local time sectors [McIlwain, 1972] and nose events [Ejiri et al., 1980].

The local time variations of the ring current particle distributions caused by changes in the magnetospheric electric fields have also been modeled. Thus, tracing equatorially mirroring protons, Chen et al. [1994] found that convective transport has a predominant role for the storm-time enhancements in the phase space densities for energies between $30-160 \mathrm{keV}$ at $L=2.5-5$. Kistler et al. [1989] and Fok et al. [1993] investigated the decay of the ring current in simple models of magnetic storms, considering drift paths of equatorially mirroring ions and different loss mechanisms along them. In this way, the former found that charge exchange is the most important loss process for the ring current population, while the latter demonstrated that Coulomb collisions between ring current ions and thermal plasma in the plasmasphere have also non negligible effects.

In the present study we take these investigations further by considering nonequatorially mirroring particles. We solve the bounce-averaged kinetic equation for arbitrary pitch angle, including losses due to charge exchange and Coulomb drag. We

*Space Physics Research Laboratory, The University of Michigan, Ann Arbor **Space Science Laboratory, NASAMSFC, Huntsville

Copyright 1994 by the American Geophysical Union.

Paper number 94GL02695

0094-8534/94/94GL-02695\$03.00 transform the ring current kinetic equation into a conservative form, and present our first results for the particle distributions.

\section{Model}

Let us consider the motion of charged particles in the magnetic field of the Earth and a superimposed electric field, which is always perpendicular to the magnetic field lines. In the guiding-center approximation, the particle's cyclotron motion is averaged out and the state of a particle is characterized by the following five variables: the three rectangular coordinates of the guiding center $x, y, z$ and its two moments $p_{I I}$ and $p_{\perp}$. The elementary volume in this fivedimensional space is [Sivukhin, 1965]:

$$
d V=p_{\perp} d x d y d z d p_{I I} d p_{\perp}
$$

Let us transform this volume element into variables $\left(R_{o}, \varphi, s, E, \mu_{o}\right)$, where $R_{o}$ is the radial distance in the equatorial plane, $\varphi$ is the geomagnetic east longitude, $s$ is the distance along the magnetic field line, $E$ is the kinetic energy of the guiding center, and $\mu_{0}=\cos \alpha_{0}$, where $\alpha_{0}$ is the equatorial pitch angle. It is possible to average out the particle's bounce motion, if we assume that the time variations of the fields are slow in comparison with the particle's bounce period (a good approximation for radial distances smaller than $10 R_{E}$ ). Integrating between the mirror points $m$ and $m^{\prime}$, we obtain the elementary volume in the four-dimensional space $\left(R_{0}, \varphi, E, \mu_{0}\right)$ :

$$
d V=R_{o}^{2} \sqrt{E} \mu_{o} f\left(\mu_{o}\right) d R_{o} d \varphi d E d \mu_{0}
$$

where $f\left(\mu_{0}\right)=\frac{1}{2 R_{0}} \int_{s_{-}}^{s_{0}} \frac{d s}{\sqrt{1-B(s) / B_{m}}}$.

In order to describe the bounce-averaged motion of the guiding centers, we first define a distribution function $Q\left(R_{0}, \varphi, E, \mu_{0}, t\right)$, such that $Q d V$ represents the mean number of particles for which the coordinates of the guiding center lie in a magnetic flux tube with equatorial area $R_{o} d R_{o} d \varphi$, and with energy and cosine of equatorial pitch angle between $E$ and $E+d E$ and $\mu_{0}$ and $\mu_{o}+d \mu_{0}$ at time $t$. We assume that the changes of the distribution function are negligible on a time scale compared with the gyro and bounce periods, which is valid in the region that our model is used.

According to the Liouville theorem, $d V=$ const and the following relation holds:

$$
\begin{array}{r}
\frac{1}{R_{o}^{2}} \frac{\partial}{\partial R_{o}}\left(R_{o}^{2}\left(\frac{d R_{o}}{d t}\right)\right)+\frac{\partial}{\partial \varphi}\left(\left\langle\frac{d \varphi}{d t}\right\rangle\right)+\frac{1}{\sqrt{E}} \frac{\partial}{\partial E}\left(\sqrt{E}\left\langle\frac{d E}{d t}\right\rangle\right) \\
+\frac{1}{f\left(\mu_{o}\right) \mu_{o}} \frac{\partial}{\partial \mu_{o}}\left(f\left(\mu_{o}\right) \mu_{o}\left(\frac{d \mu_{o}}{d t}\right)\right)=0
\end{array}
$$

The brackets $\langle$ 〉 denote bounce-averaging. Relation (3) allows us to write the bounce-averaged kinetic equation in a conservative form, which is better for numerical implementation, because it preserves the total number of particles: 


$$
\begin{aligned}
\frac{\partial Q}{\partial t} & +\frac{1}{R_{o}^{2}} \frac{\partial}{\partial R_{o}}\left(R_{o}^{2}\left(\frac{d R_{o}}{d t}\right) Q\right)+\frac{\partial}{\partial \varphi}\left(\left\langle\frac{d \varphi}{d t}\right) Q\right) \\
& +\frac{1}{\sqrt{E}} \frac{\partial}{\partial E}\left(\sqrt{E}\left(\frac{d E}{d t}\right) Q\right) \\
& +\frac{1}{f\left(\mu_{o}\right) \mu_{o}} \frac{\partial}{\partial \mu_{o}}\left(f\left(\mu_{o}\right) \mu_{o}\left(\frac{d \mu_{o}}{d t}\right) Q\right)=\left(\frac{\delta Q}{\delta t}\right)_{\text {collis }}
\end{aligned}
$$

The left hand side of this equation describes the change of the number of particles along the drift path, while the right hand side is the loss of particles due to collisions.

The bounce-averaged drift velocities of the guiding center can be obtained for the $\vec{E} \times \vec{B}$ and magnetic gradient-curvature drifts, considering that only the equatorial electric field is contributing to the bounce-averaged drift [Roederer, 1970]. The geomagnetic field in our simulation is taken to be a 3 dimensional dipole field. The electric field is expressed as the gradient of the Volland-Stem semiempirical potential model [Volland, 1973; Stem, 1975]. The radial and azimuthal bounceaveraged drift velocities are:

$$
\begin{gathered}
\left\langle\frac{d R_{o}}{d t}\right)=-\frac{A R_{o}^{\gamma+2} \cos \varphi}{M_{E}} \\
\left\langle\frac{d \varphi}{d t}\right\rangle=\frac{C+\gamma A R_{o}^{\gamma+1} \sin \varphi}{M_{E}}-\frac{3 E R_{o}}{q M_{E}} \frac{g\left(\mu_{o}\right)}{f\left(\mu_{o}\right)}
\end{gathered}
$$

Here $M_{E}$ represents the dipole moment of the Earth; $\gamma=2$; $C=1.44 \times 10^{-2} R_{E}^{2} V^{-1}$; the parameter $A$ is a function of $K_{p}$ [Maynard and Chen, 1975]; and, according to Ejiri [1978]:

$$
\frac{g\left(\mu_{o}\right)}{f\left(\mu_{o}\right)}=1-\frac{I\left(\mu_{o}\right)}{6 f\left(\mu_{o}\right)}, \text { where } I\left(\mu_{o}\right)=\frac{1}{R_{o}} \int_{s_{m}}^{s_{m}} \sqrt{1-B(s) / B_{m}} d s \text {. }
$$

The bounce-averaged rate of change of the energy of a particle of charge $q$ in a static magnetic field can be presented as the work done by the electric field on the guiding center [Northrop and Teller, 1960; Kivelson and Southwood, 1975]:

$$
\left.\left\langle\frac{d E}{d t}\right\rangle=-q \frac{\partial U}{\partial \varphi} /\left(\frac{d \varphi}{d t}\right)_{G C}\right\rangle=\frac{3 E A R_{o}^{\gamma+1} \cos \varphi}{M_{E}} \frac{g\left(\mu_{o}\right)}{f\left(\mu_{o}\right)}
$$

The bounce-averaged rate of change of the cosine of the particle's equatorial pitch angle, using the conservation of the first and the second adiabatic invariants [Ejiri, 1978], is:

$$
\left(\frac{d \mu_{o}}{d t}\right)=-\frac{\left(1-\mu_{o}^{2}\right)}{4 \mu_{o}} \frac{A R_{o}{ }^{\gamma+1} \cos \varphi}{M_{E}} \frac{I\left(\mu_{o}\right)}{f\left(\mu_{o}\right)}
$$

The important loss processes that ions undergo along their drift paths are charge exchange with neutral hydrogen from the geocorona and Coulomb collisions with thermal plasma from the plasmasphere. The charge exchange term can be written as:

$$
\left\langle\left(\frac{\delta Q}{\delta t}\right)_{c e}\right)=-\sigma_{t} \sqrt{\frac{2 E}{m_{l}}}\left\langle n_{H}\right\rangle Q
$$

where for the charge exchange cross section, $\sigma_{t}$, we used the data provided by Phaneuf et al. [1987] for $\mathrm{O}^{+}$ions, and those by Bamett [1990] for $\mathrm{H}^{+}$and $\mathrm{He}^{+}$ions. The exospheric hydrogen densities $n_{H}$ were taken from Rairden et al. [1986] and were bounce-averaged according to Schulz and Blake [1990].

In order to obtain the Coulomb drag energy degradation term, we use the Fokker-Planck equation as given by Hinton [1983]. Transforming the variables, neglecting energy diffusion and pitch-angle scattering (these are the less important loss mechanisms in the energy range considered in the model), and bounce-averaging, gives:

$$
\left\langle\left(\frac{\delta Q}{\delta t}\right)_{c c}\right\rangle=\frac{1}{\sqrt{E}} \frac{\partial}{\partial E}\left\{\sqrt{E}\left\langle\left(\frac{d E}{d t}\right)_{c c}\right) Q\right\}
$$

where $\left(\left(\frac{d E}{d t}\right)_{c c}\right)=\sqrt{E} \Gamma_{t} \sqrt{2} m_{t}{ }^{1.5} \sum_{b} \frac{\left\langle n_{b}\right\rangle Z_{b}{ }^{2}}{k T_{b}} G\left(\frac{\mathrm{v}}{\mathrm{v}_{b}}\right)$. Here the index $b$ denotes the background plasma; $v$ is the velocity of the ring current ion specie with mass $m_{t} ; v_{b}$ is the thermal velocity of $b ; \Gamma_{t}=\frac{Z_{t}^{2} e^{4} \ln \Lambda}{4 \pi \varepsilon_{o}^{2} m_{t}^{2}} ; Z_{b} e$ and $Z_{t} e$ are the charges of species $b$ and $t ; \ln \Lambda$ is the Coulomb logarithm; $\Phi$ is the error function and $G(x)=\frac{\Phi(x)-x \Phi^{\prime}(x)}{2 x^{2}}$. Assuming that the ratio $\frac{n_{b}(s)}{B(s)}$ holds constant along the magnetic field line, we obtain the bounce-averaged thermal plasma densities in the form:

$$
\left\langle n_{b}\right\rangle=\frac{n_{b o}}{1-\mu_{o}^{2}}\left[1-\frac{I\left(\mu_{o}\right)}{2 f\left(\mu_{o}\right)}\right]
$$

where the equatorial densities, $n_{b o}$, for the plasmaspheric electrons are calculated with the model of Rasmussen et al. [1993] and, those for the $\mathrm{H}^{+}, \mathrm{He}^{+}$and $\mathrm{O}^{+}$ions are estimated to be $77 \%, 20 \%$ and $3 \%$ of the electron population, respectively. The plasmaspheric temperatures are assumed to be $T_{e}=T_{i}=1 \mathrm{eV}$.

To solve numerically the bounce-averaged kinetic equation (4), we use the double splitting method, where the solution is advanced in a single dimension at each half time step. The order of the solution operators is reversed in the next half step to achieve second order accuracy in time. The Lax-Wendroff scheme and the first order upwind scheme, together with the superbee flux-limiter method are used for discretization of the drift terms and the Coulomb drag term. This is a high resolution method, which is second order accurate for smooth solutions and yet gives well resolved discontinuities [LeVeque, 1992]. An exact solution exists for the charge exchange loss term.

In the calculation presented in this paper, we used the following boundary conditions: 1) the region under consideration extends from $L=2$ to $L=6.5$, and constant injection is assumed on the nightside; 2) the kinetic energy range is between $0.01 \mathrm{keV}$ and $300 \mathrm{keV}$; 3) the solution is assumed to be periodic in azimuth; and, 4) the solution is assumed to be symmetric about $90^{\circ}$ pitch angle and zero at the atmospheric loss cone.

\section{Results}

In this paper we present preliminary results from our model. For the sake of the clarity and simplicity of our discussion, we assume time-independent electric fields, corresponding to $K_{p}=6$, and only consider the proton population, although our model is set up to also calculate $\mathrm{He}^{+}$and $\mathrm{O}^{+}$ring current distributions.

In order to study the acceleration process, when the charged particles drift adiabatically toward the Earth, we set the distribution to be initially zero everywhere except in the midnight sector, between $L=6$ and $L=6.5$, and neglect all loss processes along the drift path. The initial distribution is represented by a Gaussian with peak at magnetic midnight, at $L=6.5$, at $90^{\circ}$ pitch angle and at selected energy, with standard deviation of $10 \%$ about the peak values.

We studied the time evolution of the proton populations peaked at $0.5 \mathrm{keV}, 1 \mathrm{keV}, 10 \mathrm{keV}, 20 \mathrm{keV}$ and $50 \mathrm{keV}$, respectively. The magnetospheric regions populated by ions, as time progressed, were consistent with the ion drift paths and the numerical diffusion was reasonably small. The process of acceleration was clearly seen: the maximum of the distribution was shifted, with decreasing $L$, toward higher energies. For equatorially mirroring particles we can estimate this change in kinetic energy with $L$, applying a relation that can be derived 

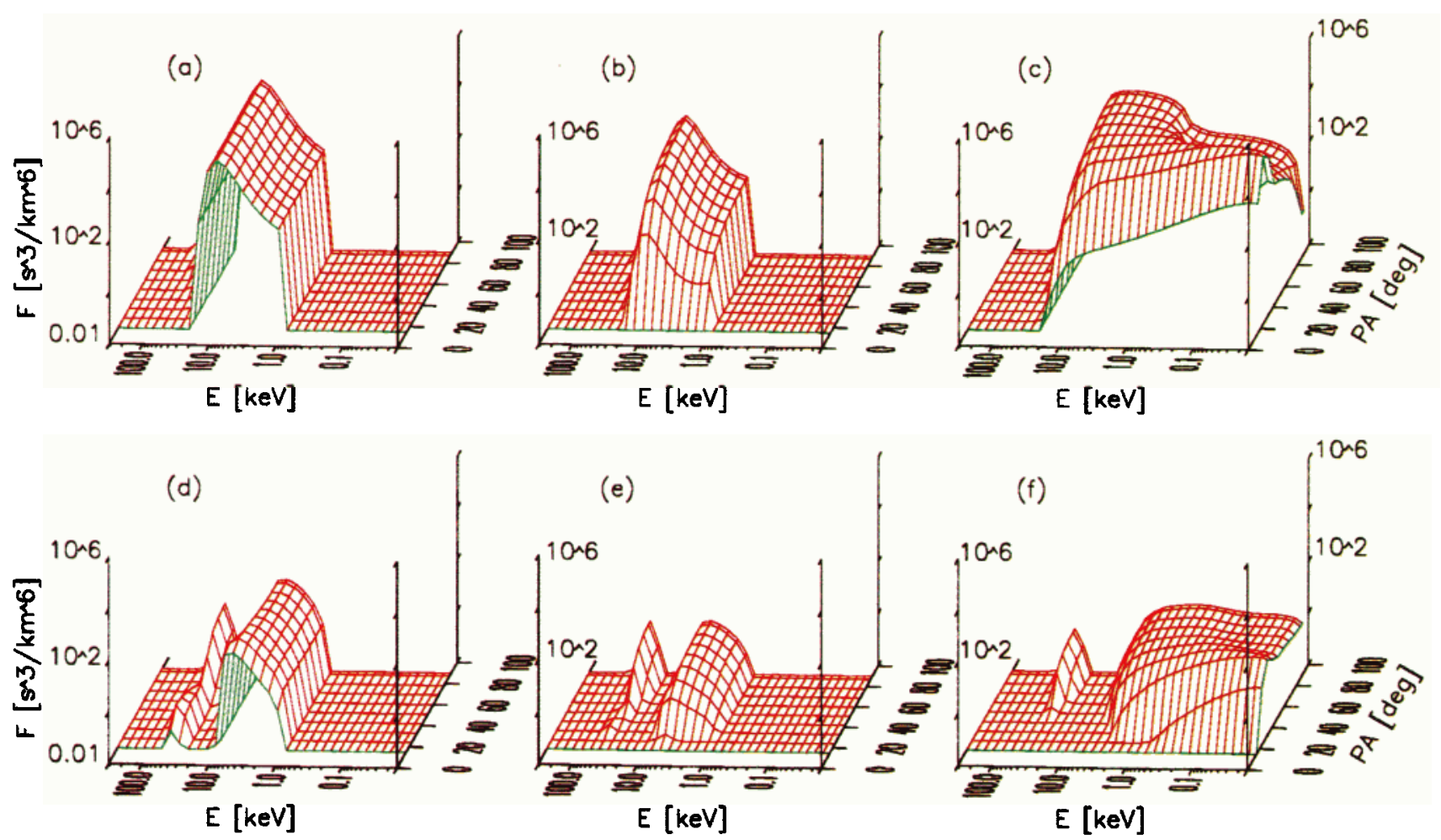

Figure 1. Velocity space distributions for $\mathrm{H}^{+}$ring current ions at $L=2.5$ : (a) initial, at $M L T=0$; (b) 9 hours from the beginning of the simulation considering charge exchange only, at $M L T=0$; (c) same as (b) but considering Coulomb drag only; (d) 9 hours from the beginning of the simulation, at $M L T=9$, considering drift only; (e) same as (d) but considering drift and charge exchange; (f) same as (d) but considering drift, charge exchange and Coulomb drag.

from (7): $d E / d R_{o}=-3 E / R_{o}$. Model results were in very good agreement with these estimates.

To study the influence of transport and loss processes on the time evolution of the distribution function, we carried out simulations with zero initial conditions everywhere except in the midnight sector between $L=2$ and $L=3$, where both the geocoronal and the plasmaspheric densities are high and the loss mechanisms are important. The maximum of the initial Gaussian distribution is located at zero magnetic local time and $L=2.5$ and is shown in Fig. 1a. The peak in energy is at $E=10$ $\mathrm{keV}$. We assumed isotropy in pitch angle, to easily compare the drift and loss effects on particles with different pitch angles. (Future studies to model specific storm-time ring current behavior will use more realistic, anisotropic, initial pitch angle distributions). The loss cone corresponds to an altitude of $1000 \mathrm{~km}$ in the atmosphere.

First, we investigate the effect of each mechanism separately. The changes in the distribution caused by charge exchange collisions between ring current protons and neutral hydrogen from the geocorona, 9 hours after the beginning of the simulation, are shown in Fig. 1b. The distribution has decreased for all energies and a strong pitch angle anisotropy has developed. The anisotropy is due to the fact that, particles with smaller pitch angle have longer bounce paths, which take them deeper into the atmosphere, where the background densities are higher, and thus undergo more collisions. Therefore, for smaller pitch angles the losses are bigger and the distribution decreases significantly. The changes in the distribution, 9 hours after the beginning of the simulation, considering Coulomb drag only, are shown in Fig. 1c. Collisions between ring current ions and thermal background plasma result in energy degradation of the high-energy ring current population and the subsequent buildup of a low-energy population. Such a buildup was modeled by Fok et al. [1993] for equatorially mirroring particles. For particles with smaller pitch angle, the energy loss proceeds faster, because, by analogy with charge exchange, such particles encounter denser plasmaspheric populations along their longer bounce paths. Therefore, the increase in the distribution at lower energies starts at smaller pitch angles and extends toward larger pitch angles with time.

To illustrate the combined effect of all these processes along the drift paths, we first present the distribution, obtained in the prenoon sector, as a result of the particles' drift motion alone (given the same initial condition as shown in Fig. 1a), and then progressively add in the effects of the loss processes. In Fig. 1d (drift only), the low-energy population has drifted eastward, while the high-energy one has moved westward. The dip in the distribution at energies of about $10 \mathrm{keV}$, is due to the small azimuthal drift velocities at these energies; the particles need more than 9 hours to reach the dayside sector. Such dips have been observed in the measured energy spectra [Mcllwain, 1972], and have been discussed for equatorially mirroring particles by previous authors [e.g. Kistler et al., 1989]. The inclusion of all pitch angles in the drift motion, shows that pitch angle anisotropies develop in the process of refilling of the "stagnation" dip. As can be seen from (6), the energy at which the azimuthal drift is about zero, decreases with increasing pitch angle. In this way, the drop off in the distribution, which occurs at about $10 \mathrm{keV}$ for $90^{\circ}$ pitch angle, shifts toward $15 \mathrm{keV}$ for smaller pitch angles. In addition, the westward drift has a maximum for $90^{\circ}$ pitch angle, while the eastward drift has a maximum for the smallest pitch angle, thus the refilling of the dip from the high-energy side and from the low-energy side proceeds from opposite pitch angle directions. All these features indicate that the drift motion is represented 
correctly in our model.

The particle distribution obtained including charge exchange losses along the ion drift paths is shown in Fig. 1e. It has significantly decreased for all energies and pitch angles, and especially for small pitch angles, where the loss mechanism is the most important. Finally, the results from our simulations, including both charge exchange and Coulomb drag losses along the drift paths are shown in Fig. 1f. When compared with the case of no collisions (Fig. 1d), the combined effect of the overall decrease in the distribution due to charge exchange and the buildup of the low-energy population due to Coulomb drag, is clearly evident.

\section{Summary}

A bounce-averaged kinetic model for arbitrary pitch angle, for $\mathrm{H}^{+}, \mathrm{He}^{+}$and $\mathrm{O}^{+}$ring current ions, was developed and solved numerically. This model is an important tool to study the pitch angle dependence of the ring current distributions. The results for the evolution of the proton population, under the conditions of time-independent electric fields, are shown, including charge exchange and Coulomb drag losses. The observed particle acceleration and pitch angle changes along the ion drift paths indicate that the drift motion and losses are correctly modeled. The effects from charge exchange and Coulomb collisions, such as a decrease of the distribution and a buildup of a low-energy population, faster for small pitch angles, are discussed.

In the near future, we plan to simulate a more complete and realistic model of a magnetic storm, considering timedependent electric fields and anisotropic initial pitch angle distributions and, to present a more detailed description of the ring current development, including the heavier ions. We also plan to add more loss mechanisms to our model, such as pitchangle diffusion due to Coulomb collisions and wave-particle interactions, which may play an important role in causing ion precipitation.

Acknowledgments. This work was supported by NASA under grant number NAGW-1619 and NSF under grand number ATM-9114409.

\section{References}

Bamett, C. F., Atomic data for fusion, vol. I, Collisions of $\mathrm{H}, \mathrm{H}_{2}$, He and $\mathrm{Li}$ atoms and Ions with atoms and molecules, Tech. Rep. ORNL. 6086/VI. Oak Ridge, Tenn., 1990.

Chen, M. W., L. R. Lyons, and M. Schulz, Simulations of phase space distributions of storm time protion ring current, L. Geophys. Res., 29 5745, 1994.

Ejiri, M., Trajectory traces of charged particles in the magnetosphere, J. Geophys. Res. 83, 4798, 1978.

Ejiri, M., R. A. Hoffman, and P. H. Smith, Energetic particle penetrations into the inner magnetosphere, J. Geophys. Res., 85, 653, 1980.

Fok, M. C., J. U. Kozyra, A. F. Nagy, C. E. Rasmussen, and G. V. Khazanov, Decay of equatorial ring current ions and associated aeronomical consequences, J.Geophys. Res, 28, 19381, 1993.
Hinton, F. L., Collisional transport in plasma, in Handbook of Plasma Bhysics, v. 1, edited by A. A. Galeev and R. N. Sudan, 163 pp., NorthHolland Publishing Co., Amsterdam, 1983.

Kistler, L. M., F. M. Ipavich, D. C. Hamilton, G. Gloeckler, B. Wilken, G. Kremser, and W. Stüdemann, Energy spectra of the major ion species in the ring current during geomagnetic storms, J. Geophys. Res. 24, 3579, 1989.

Kivelson, M. G., and D. J. Southwood, Local time variations of particle flux produced by an electrostatic field in the magnetosphere, $\underline{\mathrm{J}}$. Geophys. Res. 80, 56, 1975.

LeVeque, R. J., Numerical Methods for Conservation Laws, 2nd ed., 176 pp., Birkhäuser Verlag, Boston Mass., 1992.

Maynard, N. C., and A. J. Chen, Isolated cold plasma regions: Observations and their relation to possible production mechanisms, $\mathrm{L}$ Geophys. Res. 80, 1009, 1975.

Mcllwain, C. E., Plasma convection in the vicinity of the geosynchronous orbit, in Earth's Maqnetospheric Processes edited by B. M. McCormac, 268 pp., D. Reidel, Hingham, Mass., 1972.

Northrop, T. G., and E. Teller, Stability of adiabatic motion of charged particles in the earth's field, Phys. Rey. 117, 215, 1960.

Phaneuf, R. A., R. K. Janev, and M. S. Pindzola, Atomic data for Fusion, vol. V, Collisions of Carbon and Oxygen Ions with Electrons, $\mathrm{H}, \mathrm{H}_{2}$ and He, Tech. Rep. ORNL-6090/V5, Oak Ridge Nat. Lab., Oak Ridge, Tenn., 1987.

Rairden, R. L., L. A. Frank, and J. D. Craven, Geocoronal imaging with Dynamics Explorer, I. Geophys. Res. 21, 13613, 1986.

Rasmussen, C. E., S. M. Guiter, and S. G. Thomas, Two-dimensional model of the plasmasphere: refilling time constants, Planet. Space Sci. 41, 35, 1993.

Roederer, J. G., Dynamics of Geomagnetically Trapped Radiation 36 145 pp., Springer-Verlag, New York, 1970.

Schulz, M. and J. B. Blake, Analytical estimates for gyration-, bounce-, and drift-averaged atmospheric densities experienced by geomagnetically trapped particles (abstract), EOS Trans. AGU, 11 . 1556, 1990.

Sivukhin, D. V., Motion of charged particles in electromagnetic fields in the drift approximation, in Reviews of Plasma Physics, edited by acad. M. A. Leontovich, v. 1, 97 pp., Consultants Bureau, New York, 1965.

Stern, D. P., The motion of a proton in the equatorial magnetosphere, $J$. Geophys. Res., 80, 595, 1975.

Volland, H., A semiempirical model of large-scale magnetospheric electric fields, J. Geophys. Res., 78, 171, 1973.

Takahashi, S., and T. Iyemori, Three-dimensional tracing of charged particle trajectories in a realistic magnetospheric model, L. Geophys. Res, 94, 5505, 1989.

V. K. Jordanova, J. U. Kozyra, G. V. Khazanov, A. F. Nagy, and C. E. Rasmussen, Department of Atmospheric and Space Sciences, 2455 Hayward Street, University of Michigan, Ann Arbor, MI 48109-2143.

M.-C. Fok, NASA Marshall Space Flight Center ES-53, Bldg. 4481, Room 375A Space Science Laboratory, Huntsville, AL 35812.

(Received May 30, 1994; revised August 25, 1994; accepted September 28, 1994.) 